\title{
PERCEPÇÃO DE ESPAÇOS DE HOME OFFICE POR PORTADORES DA SÍNDROME DE BURNOUT: O ENFOQUE DA NEUROERGONOMIA PARA A AVALIAÇÃO DE LUGARES
}

\author{
Juliana Perdigão Mayer Ventura (discente) / UFPE \\ Lourival Costa Filho (orientador) / UFPE
}

\section{RESUMO}

Em anos recentes, vem se registrando um aumento no número de pessoas acometidas pela Síndrome de Burnout $(\mathrm{SB})$, doença que está associada às questões ocupacionais e que pode ser acentuada pelos lugares onde essas pessoas atuam. Diante desse contexto, utilizando conceitos e métodos da Neuroergonomia (EEG) e explorando tecnologias emergentes (realidade virtual imersiva), a pesquisa de doutorado que está em desenvolvimento no PPGDesign/UFPE, visa à apresentar uma proposta para avaliação da relação entre os indivíduos com SB e o espaço de home office, ou seja, como certas variáveis ambientais podem impactar essa relação. Intenta-se, a partir dessa compreensão, estabelecer diretrizes projetuais para a elaboração de espaços de home office que sejam capazes de proporcionar um ambiente laboral mais adequado aos usuários acometidos pela SB, tendo em vista o aumento dessa modalidade de trabalho frente à pandemia da Covid-19.

Palavras-chave: Home office; Síndrome de Burnout; Ambiente construído; Neuroergonomia; Percepção ambiental.

\section{Introdução}

A prática científica do design de interiores, entendido como um campo multidisciplinar, tem agregado novos processos visando à melhoria da qualidade de vida, do bem-estar e do conforto dos usuários dos espaços, acreditando-se que é impossível obter um ambiente adequado sem que o projeto seja fundamentado na pessoa, e leve em consideração as suas necessidades, os seus desejos e as funções a serem ali desenvolvidas.

No caso dos portadores da Síndrome de Burnout, os atributos ambientais podem exercer influência direta no quadro clínico geral associado à doença, uma vez que se tem afirmado ser ela um problema que pode ser agravado, principalmente, pelos lugares onde essas pessoas atuam (MEDLAND; HOWARD; WHITAKER, 2004).

Numa outra ponta, o advento da informatização permitiu uma maior flexibilização das relações laborais e um consequente aumento na incidência do trabalho remoto. No entanto, a pandemia da Covid-19 acelerou o processo de implantação da modalidade home office, e as mudanças de hábitos ocasionadas por essa nova realidade geram uma sobreposição de elementos estressores que podem intensificar sobremaneira as dificuldades enfrentadas pelos portadores de SB. 
Nesse sentido, é fundamental que o planejamento desses espaços tenha o usuário como elemento central do processo de projetação, levando-se em consideração não apenas as características físicoformais do ambiente, mas também observando-se e compreendendo as necessidades subjetivas (MONT'ALVÃO, 2011).

Sob essa ótica - de colocar o usuário como centro do processo de projetação -, a Neurociência aliada à Ergonomia (Neuroergonomia) tem permitido investigações mais aprofundadas sobre o ambiente construído, as preferências ambientais dos indivíduos e as respostas humanas aos estímulos gerados por esses espaços (PAIVA, 2018).

Evans e Blanke (2013) apontam para a possibilidade da combinação de Eletroencefalograma (EEG) com configurações de Realidade Virtual, a partir da qual é possível compreender as respostas humanas para as diversas possibilidades de decisão projetual, através dos dados coletados pelo biofeedback da interação cérebro-computador, possibilitando correções de inadequações antes mesmo da materialização dos espaços.

É nesse cenário que se insere a pesquisa de doutorado que está em desenvolvimento no PPGDesign/UFPE, e que é apresentada neste artigo, a partir do estágio de construção em que se encontra.

Nesse contexto, cabe destacar que, através de questionários e metodologias de percepção ambiental explorados pela Psicologia Ambiental, como a Constelação de Atributos e a Seleção Visual, objetivase, inicialmente, identificar as preferências ambientais e as qualidades afetivas mais desejáveis para um espaço de home office, visando à sensação de bem-estar físico e emocional dos indivíduos acometidos pela Síndrome de Burnout. Em uma segunda etapa, o levantamento dos biomarcadores de valência emocional e de atenção, captados através de EEG (eletroencefalografia) no momento da experiência de realidade virtual imersiva, em cenários pré-estabelecidos (realidade virtual), permitirá uma averiguação mais aprofundada e uma possível confirmação das preferências elencadas. Finalmente, a partir dos dados coletados e correlacionados, intenta-se estabelecer diretrizes projetuais para a construção e adaptação de espaços de home office adequados a esses usuários.

\section{Os espaços de home office e a percepção ambiental}

O avanço da tecnologia da informação e da comunicação (TCI) tem permitido uma maior implementação do trabalho remoto, prevendo-se que, até o ano de 2050, cerca de $50 \%$ da população ativa no mundo estará atuando em regime de home office (MORGAN, 2004). O Brasil segue essa previsão, e numa projeção intensificada pelo advento da pandemia da Covid-19, estima- 
se que já existem mais de 7 milhões de pessoas que, em algum grau, atuam nesse tipo de regime no país (IBGE, 2020).

Diversos estudos exploram as vantagens e desvantagens do trabalho remoto e analisam as inúmeras mudanças nos padrões de experiência do indivíduo com o seu ambiente físico laboral, apontando que muitos profissionais sentem a necessidade de reproduzir em casa as condições de que dispunham no âmbito da empresa (BARROS; SILVA, 2010).

Dados como esses nos direcionam para a importância de estabelecer um enfoque ergonômico sobre a situação, pois, na medida em que o espaço projetado é a interface que permite a realização das tarefas/atividades e a percepção que o indivíduo tem acerca do ambiente construído, podem determinar as suas possibilidades comportamentais (NASAR, 2000), as decisões projetuais têm um efeito importante sobre as situações de trabalho, ainda que exercidas de maneira remota.

Para Moser (2018, p. 16), "os fatores físicos e sociais estão inextrincavelmente associados em sua incidência sobre a percepção e o comportamento da pessoa", e a percepção, em si mesma, depende de características e aspirações individuais, ou seja, (KAPLAN, 1988) as experiências vivenciadas pelos indivíduos contribuem para o ajuste do padrão humano de julgamentos, sendo, portanto, a preferência, por definição, um julgamento emocional que envolve avaliação e sentimentos.

Nesse sentido, nota-se que a modulação cognitiva dos efeitos das condições ambientais desempenha um papel importante sobre a relação entre a pessoa e seu ambiente, sendo necessário compreender um conjunto de fatores psicológicos mediadores, para, então, se compreender as reações individuais (MOSER, 2018).

Segundo Villarouco e Andreto (2008), os conhecimentos sobre percepção, sensação e emoção devem ser entendidos como vitais ao projeto, aplicando-se critérios ergonômicos mais subjetivos na busca da adequação dos ambientes às necessidades dos usuários, passando a incorporar no processo de criação valores e comportamentos sociais das pessoas e dos grupos, a fim de tornar possível a criação de espaços que, realmente, contribuam para a promoção do prazer e bem-estar dos profissionais.

\section{Síndrome de Burnout e o ambiente construído}

A Síndrome de Burnout afeta hoje cerca de $30 \%$ dos brasileiros economicamente ativos, colocando o Brasil na segunda posição do ranking de países com o maior número de pessoas acometidas pela doença, superado apenas pelo Japão, onde $70 \%$ da população ativa apresenta sintomas da doença 
(ISMA-BR, 2013). Esses números crescentes levaram a Organização Mundial da Saúde a incluir o transtorno na Classificação Internacional de Doenças (CID-11), que lista enfermidades que serão prevalentes nos próximos anos (WHO, 2019).

Segundo Harrison (1999), essa síndrome pode ser considerada como um tipo de estresse agudo e de caráter duradouro, sendo resultante da constante pressão emocional associada ao intenso envolvimento laboral, por longos períodos de tempo. Além disso, autores como Medland; Howard e Whitaker (2004), têm afirmado que a referida síndrome não é exclusivamente um problema mental/psicológico - como acontece em outras doenças mentais comuns -, mas uma condição agravada também pelos lugares onde essas pessoas vivem e trabalham.

Sabemos, hoje, que a maioria das doenças mentais têm a sua base no cérebro; porém, sabemos também que essas perturbações são influenciadas por uma combinação de fatores biológicos, psicológicos, sociais e ambientais (WHO, 2002).

As pessoas passam $90 \%$ do tempo total das suas vidas em ambientes construídos e, diante da decorrência da pandemia pelo SARS-CoV2 e das mudanças de hábitos ocasionadas por ela, especialmente no que diz respeito à maior implantação da modalidade home office, esse percentual aumentou consideravelmente.

No caso dos portadores da Síndrome de Burnout, essas mudanças (menor interação interpessoal, acúmulo de atividades domésticas, escolares e de trabalho, etc) transformam-se em catalisadores dos níveis de estresse e ansiedade e podem gerar problemas ainda maiores, uma vez que, de acordo com Moreno; Gil; Haddad e Vannuchi (2011), os portadores da doença não conseguem (mesmo quando não estão trabalhando) se desassociarem mentalmente das atividades laborais; e levar o trabalho para o ambiente residencial - local preponderantemente destinado à recomposição emocional, física e psíquica - dificulta ainda mais esse processo de desassociação.

Diante disso, há um interesse crescente em investigar se, e em que medida, as variáveis que compõem o ambiente construído influenciam a prevalência desses transtornos nos indivíduos, já existindo estudos recomendando a realização de intervenções espaciais que favoreçam a interação interpessoal e que gerem melhores condições de trabalho, a partir da observância de aspectos ergonômicos (MORENO; GIL; HADDAD; VANNUCHI, 2011). No entanto, observa-se que, a maioria dos estudos tem usado dados coletados dentro dos limites socioeconômicos, bem como dos aspectos demográficos e organizacionais, havendo pouca pesquisa sobre a relação direta dos componentes físico-formais e estéticos do espaço habitável na saúde mental dos usuários, especialmente em áreas 
de pequeno e médio porte ou ambientes internos, além de focaram, em sua grande maioria, nos impactos sobre a saúde física dos ocupantes (WEICH, 2005).

\section{Neuroergonomia na identificação das preferências ambientais}

Uma vez que toda experiência humana é resultante de atividades cerebrais (OLIVEIRA, 2012) e que, como visto, a cognição e a percepção são mediadoras das atividades humanas exercidas nos ambientes físicos, a utilização de conceitos e técnicas da Neurociência tem agregado benefícios à pesquisa com abordagem no sistema ambiente-comportamento (ZEISEL, 2006).

A Ergonomia visa compreender a interação das pessoas com o ambiente e suas interfaces, e a Neurociência tem por objetivo entender como o sistema nervoso central recebe e processa informações tanto do nível celular quanto do ambiente externo que circunda uma pessoa. A Neuroergonomia, portanto, é a união dessas duas ciências, "com vistas à otimização do entendimento das estruturas e funções cerebrais, e da cognição e do comportamento humano nos ambientes de trabalho" (PAIVA, 2018, p. 120).

Desse modo, a Neuroergonomia vem suportar o processo de projetação espacial, a fim de promover uma melhor qualidade de vida aos usuários dos espaços, na medida em que contribui para o entendimento dos impactos exercidos pelos ambientes nas vivências desses indivíduos (EBERHARD, 2009).

Na busca do entendimento mais acurado sobre a interação do usuário com seu meio ambiente (entendendo aqui ser esta a reunião de espaço e artefatos que concorrem para a realização de qualquer atividade), e devido ao custo acessível da Eletroencefalografia (EEG), esse procedimento tem sido bastante utilizado para o reconhecimento do funcionamento do cérebro voltado aos processos cognitivos (SPÍNDOLA, 2010), bem como, para estudos relacionados à carga mental de trabalho, avaliação de fadiga (BALDWIN et al., 2012) e do engajamento do usuário no desenvolvimento da tarefa (VASCONCELOS, 2014).

A eletroencefalografia (EEG) é a medida neurofisiológica da atividade elétrica do cérebro usando eletrodos colocados no couro cabeludo (SANEI; CHABERS, 2007). Seu registro gráfico resultante é reconhecido por eletroencefalograma, que representa potenciais pós-sinápticos de sinal elétrico decorrente da atividade neuronal (STEINBERG, 2003).

A unidade de aquisição dos dados neurais é constituída de um módulo (touca) com canais em combinação com eletrodos (sensores) distribuídos de modo regular por áreas do couro cabeludo 
referentes aos lobos cerebrais e, atualmente, o mercado disponibiliza uma variedade de modelos de equipamentos que são utilizados em vários contextos de pesquisas.

Posicionando-se os eletrodos em áreas específicas, é possível analisar as respostas cerebrais a determinados estímulos ambientais, fornecendo condições de diferenciar quais são os estímulos estressantes ou relaxantes, por exemplo, no momento de exposição a eles.

Dessa maneira, ao associar os conhecimentos da Ergonomia aos da Neurociência, espera-se ser possível relacionar as frequências cerebrais aos comportamentos (respostas avaliativas), obtendo-se, assim, resultados mais fidedignos sobre as preferências ambientais. Ou seja, torna-se favorável compreender em que medida os atributos ambientais efetivamente influenciam as atividades cerebrais humanas e, consequentemente, a maneira que os indivíduos reagem ao/no espaço, a partir dos estímulos nele vivenciados.

\section{Considerações finais}

Este artigo pretendeu apresentar os principais aspectos teóricos e metodológicos relacionados com a tese que está em desenvolvimento no PPGDesign/UFPE, que trata da percepção dos espaços de home office por portadores da Síndrome de Burnout. Nesse sentido, pela dinamicidade desse processo construtivo e pelos limites desse tipo de comunicação, esses aspectos não podem ser considerados como conclusivos ou prontos.

No entanto, tendo em vista que a Síndrome de Burnout tem acometido, cada vez mais, pessoas em todo o mundo, e cientes de que todas as edificações possuem características físicas, químicas e biológicas que podem afetar tanto a saúde física quanto a saúde mental dos seus usuários, faz-se necessária uma melhor compreensão dos efeitos desses ambientes, nas variações da prevalência desse tipo de transtorno psíquico, bem como entender os atributos ambientais que podem contribuir, positiva ou negativamente, para os sintomas ocasionados pela referida síndrome.

Assim, para a elaboração de ambientes que promovam a qualidade em saúde, é preciso considerar e trabalhar - de forma apropriada - diversos fatores, promovendo-se uma avaliação sistêmica, ampla, apreciando-se a situação como um todo.

A Neuroergonomia, portanto, vem corroborar com esse processo, uma vez que auxilia no entendimento dos impactos exercidos pelos atributos ambientais nas reações dos indivíduos que experienciam os espaços diariamente. A partir da compreensão das preferências ambientais dos indivíduos portadores de SB, espera-se ser possível responder as hipóteses levantadas e a 
operacionalização dos objetivos traçados na pesquisa aqui comunicada, além de, em um plano mais abrangente, estabelecer diretrizes projetuais a serem aplicadas na elaboração de espaços de home office que atendam aos critérios de ergonomia do ambiente construído e que sejam capazes de promover um maior bem-estar aos usuários do espaço, a fim de contribuir efetivamente para a melhoria do quadro clínico associado à doença.

\section{Agradecimento}

A discente agradece a Professora Dra. Vilma Villarouco, por ter abraçado esse projeto e dado início à orientação desta pesquisa. Com o seu entusiasmo costumeiro e conhecimento vasto guiou os primeiros passos e, com certeza, continuará guiando de onde estiver.

\section{Referências}

BARROS, Alexandre Moço; SILVA, José Roberto Gomes da. Percepções dos indivíduos sobre as consequências do teletrabalho na configuração home-office: estudo de caso na Shell Brasil. Cad. EBAPE.BR [online]. 2010, vol.8, n.1, pp.71-91. ISSN 1679-3951.

EBERHARD, John Paul. Brain landscape: the coexistence of neuroscience and architecture New York: Oxford University Press, Inc., 2009.

EVANS, Nathan; BLANKE, Olaf. Shared electrophysiology mechanisms of body ownership and motor imagery. Neuroimage, v. 64, p. 216-228, 2013.

HARRISON, B. J. Are you to burn out? Fund Raising Manage 1999.

ISMA-BR. INTERNATIONAL STRESS MANAGEMENT ASSOCIATION. Burnout: $30 \%$ sofrem do tipo de estresse mais devastador. 2013. Disponível em: <http://www.ismabrasil.com.br/artigo/burnout-y-30sofrem-do-tipo-de-estresse-mais-devastador>. Acesso em: 8 de jul. 2021.

KAPLAN, S. Perception and landscape: conceptions and misconceptions. In NASAR, J. L. (Ed.). Environmental Aesthetics: theory, research, and application. New York: Cambridge University Press, 1988. p. 45-55;

MEDLAND J, HOWARD RJ, WHITAKER E. Foresting psychosocial wellness oncology nurses: addressing burnout and social support in the workplace. Oncol Nurse Forum. 2004; 31(1): P. 47-4.

MONT'ALVÃO, Claudia, A ergonomia do ambiente construído no Brasil. In MONT'ALVÃO, Claudia; VILLAROUCO, Vilma (org). Um novo olhar para o projeto: A ergonomia no ambiente construído. Volume 1. Rio de Janeiro: 2AB, 2011. p. 13-24.

MORENO, Fernanda Novaes; GIL, Gislaine Pinn; HADDAD, Maria do Carmo Lourenço; VANNUCHI, Marli Terezinha Oliveira. Estratégias e intervenções no enfrentamento da Síndrome de Burnout. Rev. enferm. UERJ, Rio de Janeiro, jan/mar; 19(1):140-5, 2011. Disponível em: http://ead.bauru.sp.gov.br/efront/www/content/lessons/76/TExto\%20complementar\%20\%20estrat\%C3\%A9gias\%20e\%20interven\%C3\%A7\%C3\%B5es\%20no\%20enfrentamento\%20da\%20S\% C3\%ADndrome\%20de\%20Burnout.pdf. Acesso em: jan./2020.

MORGAN, R. Teleworking: an assessment of the benefits and challenges. European Business Review, v.16, n.4, p.344-357, 2004. 
MOSER, Gabriel. Introdução à Psicologia Ambiental: pessoa e ambiente. Campinas, SP: Editora Alínea, 2018.

NASAR, J. L. The evaluative image of places. In: WALSH, W. B.; CRAIK, K. H.; PRINCE, R. H. 2nd. ed. (Eds.). Person environment psychology: new directions and perspectives. New Jersey: Lawrence Erlbaum Associates, p. 117-168. 2000.

OLIVEIRA, Ana Beatriz A. Luz - Elo entre Neurociência e Arquitetura. IPOG. Revista Especialize on line. Edição no3, maio 2012.

PAIVA, Marie Monique Bruère. Percepção de salas residenciais por idosos - uso das técnicas de Seleção Visual, Realidade Virtual e Eletroencefalografia. Tese (Doutorado em Design). Universidade Federal de Pernambuco, Recife, 2018.

PENARANDA, B. N.; BALDWIN, Carryl L. Temporal factors of EEG and artificial neural network classifiers of mental workload. In: Proceedings of the Human Factors and Ergonomics Society Annual Meeting. Sage CA: Los Angeles, CA: SAGE Publications, 2012. p. 188-192.

SANEI, Saeid \& CHABERS, J. A. EEG Signal Processing. Chichester | England: John Wiley \& Sons, 2007.

SPÍNDOLA, Marilda Machado. Habilidade cognitiva especial: medida com eletroencefalografia. Tese (Doutorado em Informática na Educação). Universidade Federal do Rio Grande do Sul, Porto Alegre, 2010.

STEINBERG, Robert. Cognitive Psychology. 3tr ed. Belmont | CA | USA: Thomson Wadsworth, 2003.

VILLAROUCO, V.; ANDRETO, L. F. M. Avaliando desempenho de espaços de trabalho sob o enfoque da ergonomia do ambiente construído. Produção, v. 18, n. 3, p. 523-539, 2008.

WEICH, Scott. Absence of spatial variation in rates of the common mental disorders. Journal of Epidemiology \& Community Health, v. 59, p. $254-257,2005$.

WHO. World Health Organization. Depression and Other Common Mental Disorders: Global Health Estimates. Geneva, 2017. Licence: CC BY-NC-SA 3.0 IGO. Disponível em:<

https://www.who.int/publications/i/item/depression-global-health-estimates>. Acesso em: 15 de março de 2021.

WHO. World Health Organization. ICD-11 implementation or transition guide. Geneva: WHO; 2019 [cited 2019 Aug 20]. License: CC BY-NC-SA 3.0 IGO. Disponível em: https://icd.who.int/docs/ICD11\%20Implementation\%20or\%20Transition\%20Guide_v105.pdf. Acesso em: 12 de junho de 2021.

WHO. World Health Organization. Relatório Mundial da Saúde - Saúde mental: nova concepção, nova esperança. Geneva, 2002.

ZEISEL, John. Inquiry by design: environment/ behavior/ neuroscience in archtecture, interiors, landscape, and planning. New York: W. W. Norton; Revised edition, 2006. 